\title{
The Next Generation of Cosmic-Ray Experiments at the Highest Energies
}

\author{
J. Matthews \\ Department of Physics, University of Michigan \\ Ann Arbor, MI 48109
}

\begin{abstract}
A workshop was held in April 1992 in Paris to gather ideas on what will be required to properly investigate the highest energy cosmic rays. We present here a very brief summary of some of these discussions. The complete Proceedings of the workshop, containing both theoretical work and specific experimental proposals, have recently been published [1].
\end{abstract}

\section{Physics Issues.}

The energy spectrum of cosmic rays exhibits a steep power law behavior and has several prominent features. The most apparent is the sudden change in spectral index near $10^{15} \mathrm{eV}$. There may be further structure at the highest energies. The nature of these effects is entirely uncertain. While the break at the "knee" has been observed by several groups, the spectrum above $10^{19} \mathrm{eV}$ has been measured with much less precision. The flux at the highest energies is so small that only about 1000 events have been collected over thirty years of observations. Direct measurement of the elemental composition (by balloons and satellites) also suffers from low fluxes. We know the isotopic abundances of cosmic rays only up to a few hundred $\mathrm{TeV}$.

The basic questions of cosmic ray physics thus remain unanswered at the highest energies: what are the primary particles, where do they come from, and how do they attain such extraordinary energies? Below the knee, it is likely that cosmic radiation is created in supernovae and accelerated, at least in part, by supernovae blast waves. Beyond this energy, it not even clear whether the particles are originating within our own Galaxy.

All models of cosmic ray origin must be tested against the facts. Regardless of its details, any successful theory must address the energy spectrum, composition, and directionality (i.e., isotropy over the sky) or lack thereof. It therefore useful to consider in general terms how the next generation of experiment will measure these quantities. Specific propositions for a general theory of cosmic rays will provide detailed predictions for experiments, but any experiment must at least provide a thorough data set of the above three quantities.

The $\sqrt{s}$ in the interaction of of a $10^{19} \mathrm{eV}$ proton colliding with an atmospheric nucleus is more than $100 \mathrm{TeV}$. Cosmic radiation provides the only access to this extreme regime of particle physics, and will continue to do so even after the commissioning of the SSC. 
Three issues influence our thinking as we design an experiment: 1) The energy spectrum of those cosmic rays which are of extra-galactic origin will likely cut off abruptly above $\sim 10^{20} \mathrm{eV}$, the photoproduction threshold for protons interacting with the cosmic microwave background; 2) The composition of cosmic rays produced in the Galaxy will begin to become heavier at high energies as the lighter particles begin to escape; 3 ) the radius of curvature of charged particles in typical interstellar or intergalactic magnetic fields will become very large at the highest energies so cosmic rays will begin to "point back" to their source.

\section{Technology.}

Our benchmarks for the next generation of experiments will be an acceptance of $>10^{4} \mathrm{~km}^{2} \mathrm{sr}$ and the ability to reconstruct the primary energy and direction with the high accuracy. We consider extensions of the current technologies and what improvements will be required.

There are now two kinds of experiments which measure the highest energy cosmic rays. Air shower arrays measure the arrival times and numbers of the particles resulting from the interaction of the primary with the atmosphere. Optical devices, notably the Fly's Eye experiment, measure the atmospheric scintillation and flourescence excited by the air shower as it progresses.

Surface arrays reconstruct the direction of the primary very accurately and remain operational 24 hours a day. The muons and electrons can be separately measured, giving information on the nature of the primary. They can only measure the energy indirectly by reconstructing the size of the shower and then using monte-carlo simulation in order to estimate the energy.

Optical devices perform more like calorimeters as they observe the total integrated energy loss in the atmosphere. Since the air shower need not land on the device, the target area is very large. These experiments are usually limited to operating on dark, moonless nights and require a relatively clear atmosphere, so the fraction of the time the device is operable is only about $10 \%$. It will clearly be desirable to use both approaches, optical and array, in the next generation of experiments.

A typical design for the air-shower array part of the facility involves stations spaced approximately $1.5 \mathrm{~km}$ apart on a square grid. A station is composed of several scintillators and would trigger itself and store pulse height and arrival times. The stations either report their information to or are polled by a central facility. Connection to the center is made perhaps by fiber optics (probably very expensive) or by microwave radio links. Cost estimates are in the vicinity of 25-50 $\mathrm{M} \$$ for this sort of design.

The Hi-Resolution Fly's Eye (currently under construction) provides a paradigm for an optical device. The major expense is for many high quality photomul- 


\section{Next Generation of Cosmic-Ray Experiments}

tipliers. Several nearby, but separate sites can cover a similar or larger target area as an array for roughly the same cost.

The technology appears well known at this time. Widely separated installations can be powered by solar-cell batteries in the same manner that is currently used in remote civil engineering applications. Cellular telephones illustrate one contemporary example of radio communications with sufficient bandwidth. Ordinary scintillators are sufficient for air shower arrays and the Fly's Eye technology has been succesfully employed.

Because showers at these energies are so large, the altitude of the experiment is not a critical concern. Locations in the USA, South America, and Australia have been identified as suitable. A southern Hemisphere locale would provide a good "view" of the Galactic Center.

\section{Summary.}

We conclude that the next generation of experiment appears quite feasible as an expansion of current techniques. As summarized by Alan Watson: "We know what needs to be done and how to do it. It only requires money and will."

\section{REFERENCE}

1. Proceedings of the International Workshop on Techniques to Study Cosmic Rays with Energies Greater than $10^{19} \mathrm{eV}$ (M. Boratav, J.W. Cronin, and A.A. Watson, eds.), Nucl. Physics B (Proc. Suppl.) 28B (1992). 